\title{
Diagnostic advances and opportunities in chronic thromboembolic pulmonary hypertension
}

\author{
Andrea M. D’Armini
}

Affiliation: Dept of Cardio-Thoracic and Vascular Surgery, Heart and Lung Transplantation and Pulmonary Hypertension Unit, Foundation "I.R.C.C.S. Policlinico San Matteo", University of Pavia School of Medicine, Pavia, Italy.

Correspondence: Andrea M. D’Armini, Dept of Cardio-Thoracic and Vascular Surgery, Heart and Lung Transplantation and Pulmonary Hypertension Unit, Foundation "I.R.C.C.S. Policlinico San Matteo", University of Pavia School of Medicine, Viale Golgi 19, I-27100 Pavia, Italy. E-mail: darmini@smatteo.pv.it

ABSTRACT Chronic thromboembolic pulmonary hypertension (CTEPH) is characterised by the presence of thromboembolic material in the pulmonary circulation, and patients have a poor prognosis without treatment. Patients present with nonspecific symptoms, such as breathlessness and syncope, which means that other more common conditions are sometimes suspected before CTEPH, leading to delayed diagnosis and treatment. This is problematic because CTEPH is potentially curable with surgical pulmonary endarterectomy (PEA); indeed, CTEPH should always be considered in any patient with unexplained pulmonary hypertension $(\mathrm{PH})$.

Several key evaluations are necessary and complementary to confirm a diagnosis of CTEPH and assess operability. Echocardiography is initially used to confirm a general diagnosis of $\mathrm{PH}$. Ventilation/perfusion scanning is then essential in the first stage of CTEPH diagnosis, with a wedge-shaped perfusion deficit indicative of CTEPH. This should be followed by right heart catheterisation (RHC) which is mandatory in confirming the diagnosis and providing haemodynamic parameters that are key predictors of the risk associated with PEA and subsequent prognosis. RHC is ideally coupled with conventional pulmonary angiography, the gold-standard technique for confirming the location and extent of disease, and thus whether the obstruction is surgically accessible. Computed tomographic pulmonary angiography is also now routinely used as a complementary technique to aid diagnosis and operability assessment.

Recent improvements in the resolution of other noninvasive techniques, such as cardiac magnetic resonance imaging, allow for detailed reconstructions of the vascular tree and imaging of vessel defects, and interest in their use is increasing.

@ERSpublications

A review of established techniques and alternative noninvasive methods for the diagnosis and evaluation of CTEPH http://ow.ly/Lxzr4

\section{Introduction}

As with other forms of pulmonary hypertension $(\mathrm{PH})$, patients with chronic thromboembolic pulmonary hypertension (CTEPH) present with progressive dyspnoea on exertion, fatigue and possibly syncope, haemoptysis, and signs of right heart failure [1]. Symptoms tend to occur only after more than $40 \%$ of the vascular bed has been obstructed [2]; indeed, the disease is often characterised by an initial symptom-free "honeymoon period" [3]. The nonspecific symptoms of CTEPH mean that other more common

Received: Feb 062015 | Accepted after revision: April 092015

Conflict of interest: Disclosures can be found alongside the online version of this article at err.ersjournals.com

Provenance: Publication of this peer-reviewed article was sponsored by Bayer Pharma AG, Berlin, Germany (principal sponsor, European Respiratory Review issue 136).

Copyright OERS 2015. ERR articles are open access and distributed under the terms of the Creative Commons Attribution Non-Commercial Licence 4.0. 
conditions, such as asthma, chronic obstructive pulmonary disease (COPD) and general deconditioning, are often suspected before CTEPH.

The most common cause of CTEPH is acute pulmonary embolism (PE), with an estimated prevalence of $0.8-3.8 \% 2$ years after the occurrence of acute PE $[4,5]$. Other risk factors for the development of CTEPH include splenectomy, infected pacemakers and ventriculoatrial shunts $[6,7]$. Although PE is the primary cause of CTEPH, up to $62 \%$ of patients with proximal disease and $49 \%$ of those with distal disease have no history of acute PE [8], which can delay diagnosis in some cases.

These challenges to effective and rapid diagnosis are particularly problematic because CTEPH is potentially curable by surgical pulmonary endarterectomy (PEA), but carries a poor prognosis if treatment is not initiated early. The median time between symptom onset and PEA is $~ 1.5$ years [9]. Delays in diagnosis can lead to the development of more distal vasculopathy and right heart dysfunction as a result of elevated pulmonary artery pressure (PAP). Historically, 3-year mortality rates as high as $90 \%$ have been reported in patients with PAP $>50 \mathrm{mmHg}$ [10]. However, following PEA, a 3-year survival rate of $91.2 \%$ has been reported [11] and in-hospital mortality rates are $<5 \%$ in specialist centres [1]. In addition, the right ventricle is able to reversibly remodel, even in patients with significantly impaired right ventricular function before surgery $[12,13]$. Thus, it is crucial that diagnosis and treatment are initiated early and all patients with a confirmed diagnosis of CTEPH should be assessed for operability at a specialist centre [14].

Importantly, although PEA is the gold-standard treatment for CTEPH, not all patients are suitable for PEA. Thromboembolic disease located in smaller, more distal vessels may be regarded as inaccessible, depending on the experience of the surgeon, and/or comorbidities may preclude surgery [14]. Patients deemed inoperable or those who have persistent or recurrent PH after PEA may be candidates for targeted medical therapy $[1,14]$. Pharmacological treatment of CTEPH is reviewed elsewhere in this issue of the European Respiratory Review [15]. In addition, balloon pulmonary angioplasty is emerging as a potential new treatment for selected inoperable patients with CTEPH [16].

\section{Diagnostic techniques for the confirmation of CTEPH}

Several imaging modalities (table 1), outlined in detail below, are necessary and complementary to diagnose CTEPH, evaluate functional and haemodynamic impairment, and assess operability [14]. These diagnostic tests should be performed in a stepwise process (fig. 1). Echocardiography is one of the initial tests to confirm a general diagnosis of $\mathrm{PH}$, which is then followed by ventilation/perfusion $\left(V^{\prime} / \mathrm{Q}^{\prime}\right)$ scanning. An initial CTEPH diagnosis based on $V^{\prime} / Q^{\prime}$ scanning should then be confirmed by a haemodynamic evaluation using right heart catheterisation (RHC). RHC should ideally be coupled with conventional pulmonary angiography, which is the gold-standard technique for assessing the location and extent of thromboembolic disease, and therefore whether a patient is suitable for PEA. Computed tomographic pulmonary angiography (CTPA) is also routinely used to provide additional data to support CTEPH diagnosis and operability assessment. Figure 2 illustrates images from $V^{\prime} / Q^{\prime}$ scanning, conventional pulmonary angiography and CTPA. In addition, improved resolution in other imaging modalities, such as magnetic resonance pulmonary angiography (MRPA) and dual-energy computed tomography angiography (DECTA), has led to increased interest in their use in the evaluation of patients with CTEPH before PEA.

\section{Preliminary diagnostic assessments}

Given the nonspecific nature of symptoms in patients first presenting with CTEPH, more common conditions, such as COPD, asthma and simply deconditioning, are often suspected. Patients are, therefore, often first evaluated with chest radiography, pulmonary function testing, biomarker analyses, echocardiography and/or ECG. The results of these tests can suggest $\mathrm{PH}$, but are not specific for the diagnosis of CTEPH, which can result in misdiagnosis.

In the early stages of CTEPH, the chest radiograph is likely to appear normal. As the disease progresses, typical signs of $\mathrm{PH}$ can be observed including dilatation of the main, right and left pulmonary arteries as well as cardiomegaly [17]. Pulmonary function tests in patients with CTEPH are usually unremarkable although in some patients the diffusing capacity of the lung for carbon monoxide may be reduced and a mild restrictive pattern may be seen in some patients with parenchymal scarring [20]. Generally, pulmonary function testing is used to rule out other parenchymal lung diseases or airflow obstruction.

Blood tests, immunology and abdominal ultrasound scans are sometimes also used to aid diagnosis of CTEPH. It is typical to find no significant biochemical or haematological changes in routine blood tests of patients with CTEPH; however, lupus anticoagulant/anti-cardiolipin antibodies and increased levels of Factor VIII have been reported in a proportion of patients with CTEPH [21, 22]. Brain natriuretic peptide (BNP) is commonly used as a biomarker for CTEPH. BNP levels have been shown to correlate with right ventricular remodelling and identify right ventricular dysfunction [23], and normal N-terminal proBNP 
TABLE 1 Summary of techniques used in the diagnosis of chronic thromboembolic pulmonary hypertension (CTEPH)

Technique

Ventilation/perfusion

$\left(V^{\prime} / Q^{\prime}\right)$ scan

\section{Conventional pulmonary angiography (CPA)}

Right heart catheterisation (RHC)

\section{Computed tomography pulmonary angiography (CTPA)}

\section{Cardiac magnetic resonance imaging (MRI)}

Advantages

Potential disadvantages

Essential for diagnosing CTEPH

Sensitivity $>96 \%$

Negative result rules out $\mathrm{CTEPH}$

Can distinguish between large-vessel occlusive and small-vessel pulmonary vascular disease

Less radiation exposure than CTPA

Less likely than other techniques to detect incidental findings

Avoids potential problems with intravenous contrast

Can be combined with $\mathrm{RHC}$ to provide imaging and haemodynamic information

Mandatory in diagnosing CTEPH

Essential measurements of mean arterial pressure and pulmonary capillary wedge pressure

Information on disease severity, right heart function, mixed venous oxygen saturation

\section{Noninvasive}

High-resolution images

New scanners can provide multi-planar and three-dimensional reconstructions of the vascular tree More anatomical detail than MRI: information about vascular wall thickness and surrounding structures not appreciable with CPA

No need for direct catheter access Can reveal associated findings, e.g. bronchial artery collaterals, mosaic perfusion patterns Lower cost than CPA

Rapid acquisition, even in breathless patients DECTA has improved the detection of distal CTEPH

Noninvasive, no radiation exposure Morphological, functional and anatomical assessment of heart and pulmonary circulation Useful for repeat studies, e.g. pre- and post-operative monitoring

Phase-contrast MRI can quantify blood flow and peak velocity in the main pulmonary artery

Contrast-enhanced magnetic resonance angiography has similar sensitivity to CTPA
Can give intermediate probability result, which cannot rule out $\mathrm{CTEPH}$ May underestimate the burden of vascular obstruction

Invasive

Invasive

Sensitivity $51 \%$ (lower than $V^{\prime} / Q^{\prime}$ scan) Chronic disease looks different to acute PE: additional training required

Risk of false positives (e.g. pulmonary artery sarcomal

May miss disease in distal segmental or subsegmental vessels

May miss inoperable patients who could begin medical therapy

May underestimate clot burden

Limited availability, expensive, time consuming

levels in conjunction with ECG characteristics have been proposed as "CTEPH rule-out criteria" following acute PE [24]. Other proteins, such as C-reactive protein, heart-type fatty acid-binding protein and recently pentraxin 3 , have also been reported as potential biomarkers for CTEPH [25-27], although their diagnostic potential has not been systematically studied.

In terms of ECG findings, right ventricular hypertrophy is present in $87 \%$ of patients with idiopathic pulmonary arterial hypertension (PAH), and right axis deviation is present in 79\% [28]. In a study of 112 patients with confirmed CTEPH who underwent ECG, $62 \%$ presented with negative T waves V1-V4 and $50 \%$ with right axis deviation at baseline [29]. Therefore, ECG may provide suggestive or supportive evidence of $\mathrm{PH}$, but has insufficient sensitivity (55\%) and specificity (70\%) to be used as a screening tool for $\mathrm{PH}[19]$.

Echocardiography uses Doppler ultrasound to estimate pulmonary artery systolic pressure and provides a simple noninvasive assessment of cardiac structure and function. The tricuspid regurgitation velocity and estimated pulmonary artery systolic pressure are usually increased in patients with CTEPH, with defined criteria of $>2.8 \mathrm{~m} \cdot \mathrm{s}^{-1}$ and $>36 \mathrm{mmHg}$, respectively, indicating the possibility of $\mathrm{PH}$ [30]. Right 


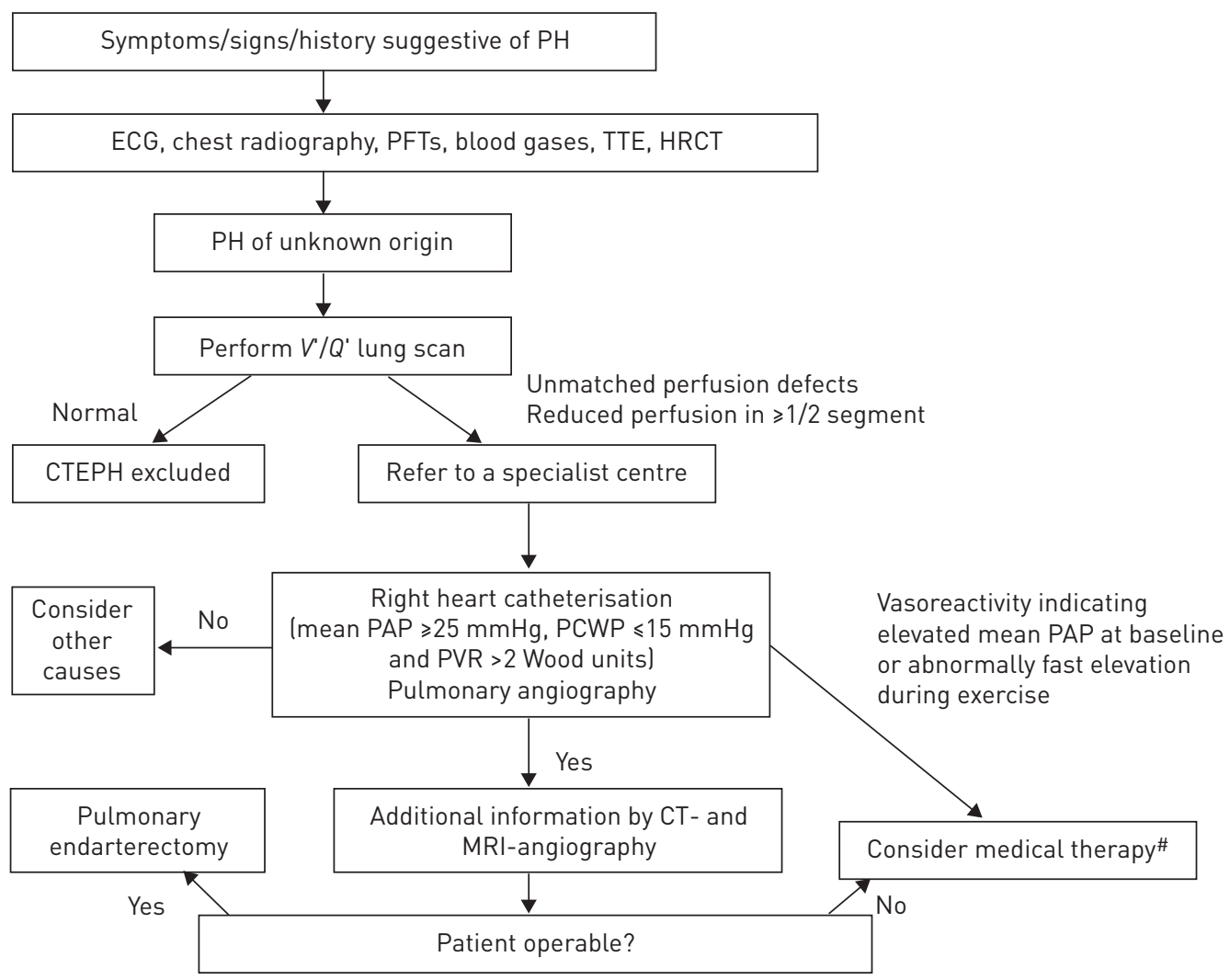

FIGURE 1 Diagnostic algorithm for patients with chronic thromboembolic pulmonary hypertension (CTEPH) [14, 19]. PH: pulmonary hypertension; PFT: pulmonary function test; TTE: transthoracic echocardiography; HRCT: high-resolution computed tomography; $V^{\prime} / Q^{\prime}$ : ventilation/perfusion; PAP: pulmonary artery pressure; PCWP: pulmonary capillary wedge pressure; PVR: pulmonary vascular resistance; CT: computed tomography; MRI: magnetic resonance imaging. ${ }^{*}$ : riociguat is currently the only licensed therapy for CTEPH.

atrial enlargement and right ventricular hypertrophy with reduced systolic function may also be detected [31]. In some cases, leftward displacement of the septum, which can lead to left ventricular dysfunction, is also observed [31]. The use of three-dimensional echocardiography can improve right ventricular assessment.

Follow-up echocardiography in patients with acute PE showing signs of $\mathrm{PH}$ or right ventricular dysfunction can determine whether $\mathrm{PH}$ has resolved. However, the systematic follow-up of patients with acute PE is a matter of debate [1]. Despite the important risk of developing CTEPH after acute PE, the overall risk is low in the majority of cases, so routine screening of asymptomatic patients is not currently recommended [32]. However, vigilance is required in patients who remain symptomatic after acute PE, and appropriate follow-up echocardiography should be considered in these patients [1]. Once PH is suspected, CTEPH should always be considered, particularly in patients with a history of, or risk factors for, chronic thromboembolic disease [19].

\section{Ventilation/perfusion scintigraphy}

Diagnostic algorithms consistently recommend the use of radionuclide $V^{\prime} / Q^{\prime}$ lung scanning to screen for thromboembolic disease $[19,33]$, and this technique is the preferred and recommended step in the initial diagnosis of CTEPH [14]. $V^{\prime} / Q^{\prime}$ scanning utilises scintigraphy and medical isotopes to evaluate the circulation of air and blood in the lungs. A wedge-shaped perfusion deficit with a normal ventilation scan is typical of CTEPH (fig. 2a) [34], while the complete absence of perfusion to one lung could be indicative of other conditions (e.g. malignancy or vasculitis) [18]. A normal perfusion scan or the presence of small peripheral unmatched and nonsegmental defects in perfusion increases the likelihood of PAH [35]. This enables a differentiation between CTEPH and PAH (the most likely alternative diagnostic possibility) to be made [14], thus providing the impetus for further diagnostic tests to be performed.

Importantly, the $V^{\prime} / Q^{\prime}$ scan has a negative predictive value of $\geqslant 98 \%$, a sensitivity rate of $\geqslant 96 \%$ and specificity rate of $\geqslant 90 \%$ for the detection of CTEPH, meaning that CTEPH can effectively be ruled out if the scan is normal [36]. In the prospective international CTEPH registry, $99.4 \%$ of patients had evidence of 
a)
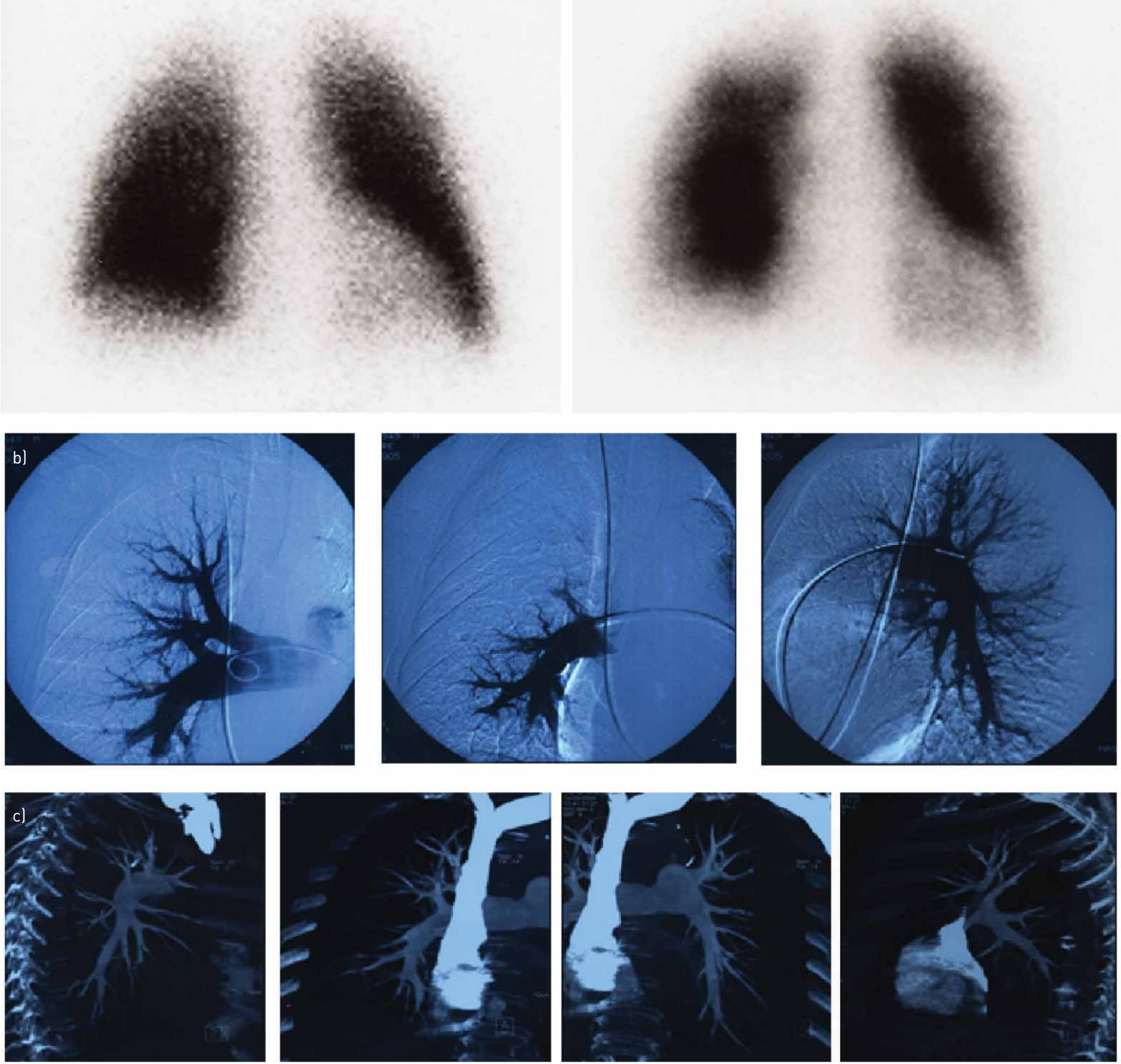

FIGURE 2 Diagnostic imaging of distal chronic thromboembolic pulmonary hypertension lesions. a) Ventilation/perfusion scintigraphy. b) Conventional pulmonary angiography. c) Computed tomography pulmonary angiography.

perfusion abnormalities on $V^{\prime} / Q^{\prime}$ scan [9]. This is one of the main reasons why the $V^{\prime} / Q^{\prime}$ scan is the preferred screening test for CTEPH over other imaging techniques such as CTPA, which has a lower sensitivity in detecting CTEPH. A retrospective review of $V^{\prime} / Q^{\prime}$ scanning and CTPA results from patients referred to a $\mathrm{PH}$ centre demonstrated sensitivity rates of $\geqslant 96 \%$ and $51 \%$, respectively, in detecting CTEPH [36]. The $V^{\prime} / Q^{\prime}$ scan also has a higher sensitivity in detecting very distal forms of the disease compared with CTPA, so disease confined to such areas can be missed if screened by CTPA rather than a $V^{\prime} / Q^{\prime}$ scan [14]. Furthermore, the $V^{\prime} / Q^{\prime}$ scan is easier to interpret than CTPA as the defects after PE or with chronic disease usually appear identical on a $V^{\prime} / Q^{\prime}$ scan, while defects associated with chronic disease look different with CTPA $[37,38]$. This makes the $V^{\prime} / Q^{\prime}$ scan a reliable screening technique for diagnosis in all centres. 
However, $V^{\prime} / Q^{\prime}$ scanning is not without pitfalls. For example, it may underestimate the extent of central vascular obstruction [39], and other conditions, such as fibrosing mediastinitis and pulmonary veno-occlusive disease, can also present as segmental perfusion defects on $V^{\prime} / Q^{\prime}$ scanning [18]. Therefore, additional imaging techniques are required in patients with suspected CTEPH in order to definitively rule out other conditions [34].

Although the current CTEPH diagnostic algorithm recommends $V^{\prime} / Q^{\prime}$ scanning [14], evidence suggests that $V^{\prime} / Q^{\prime}$ scans are not widely used in the evaluation of patients with unexplained $\mathrm{PH}$, which may contribute to underdiagnosis of CTEPH. Recent data from a $\mathrm{PH}$ registry showed that $43 \%$ of patients diagnosed with $\mathrm{PAH}$ never underwent a $V^{\prime} / Q^{\prime}$ scan leading up to their diagnosis [40]. Bearing in mind the lower sensitivity of other imaging techniques, such as CTPA, in detecting CTEPH, it is possible that some patients presumed to have PAH may actually have CTEPH [14]. It was also reported that close to one-third of these patients were not given a $V^{\prime} / Q^{\prime}$ scan because the provider thought it was not relevant, thus highlighting the need for improved education about CTEPH for physicians [14]. It is, therefore, essential that all patients with suspected CTEPH be referred to a specialist centre for a complete diagnostic evaluation to exclude or confirm the diagnosis and assess operability $[14,19]$.

\section{Right heart catheterisation}

If a $V^{\prime} / Q^{\prime}$ scan suggests CTEPH, patients should be referred to a specialist centre for RHC and pulmonary angiography. Preferably, if logistically possible, RHC should be carried out at the same time as pulmonary angiography at a centre that is capable of assessing patients for PEA to avoid repeating these invasive procedures [1]. Haemodynamic evaluation via RHC is mandatory in diagnosing CTEPH as it assesses the severity of the haemodynamic impairment, and haemodynamic parameters are key predictors of prognosis and the risk associated with PEA $[14,18]$. The presence of pre-capillary PH (mean PAP $\geqslant 25 \mathrm{mmHg}$, pulmonary capillary wedge pressure $\leqslant 15 \mathrm{mmHg}$ and pulmonary vascular resistance (PVR) $>2$ Wood units) in patients with chronic/organised thromboembolic obstructions confirms the final diagnosis of CTEPH [19].

\section{Pulmonary angiography}

Conventional angiography

Catheter-based selective pulmonary angiography, with digital subtraction angiography to improve vessel contrast especially in the distal arteries, is considered the gold-standard diagnostic tool for confirmation of CTEPH (fig. 2b) [14]. It is also used to determine the extent and distribution of disease thereby providing information regarding the patient's eligibility for PEA. A single injection of non-ionic contrast is injected into both proximal pulmonary arteries, with the amount of contrast adjusted according to the patient's cardiac output, and ideally biplane images are taken [20].

Typical angiographic defects present in CTEPH correspond to the organisation and recanalisation that follows an acute thromboembolic event. These include pulmonary artery webs or bands, intimal irregularities, abrupt narrowing of the pulmonary arteries, proximal obstruction and pouch defects [20]. These defects are very distinct from those observed in acute PE, where well-defined intraluminal filling defects are observed.

One major advantage of the catheter-based pulmonary angiogram is that it can be combined with RHC to assess haemodynamic parameters [14]. The radiographic and haemodynamic data are complementary in determining a patient's suitability for PEA, as well as predicting perioperative risk [41].

\section{Computed tomography pulmonary angiography}

CTPA is used in the differential diagnosis of CTEPH and as part of the routine assessment for operability, complementing $V^{\prime} / Q^{\prime}$ scans and pulmonary angiography. It is also indicated when a $V^{\prime} / Q^{\prime}$ scan is indeterminate or shows perfusion defects. CTPA can provide a multi-planar, three-dimensional reconstruction of the vascular tree (fig. 2c). Multi-detector CTPA provides resolution approaching $0.5 \mathrm{~mm}$ in all planes and permits rapid acquisition, requiring only 5-10 s of breath-hold time, allowing examination of even critically ill or dyspnoeic patients [34, 42, 43]. The sensitivity and specificity for detection of thromboembolic disease with 64 - or 320-detector row CTPA is $97.0-98.3 \%$ and $94.8-97.1 \%$ at the lobar level, and $85.8-94.1 \%$ and $92.9-94.6 \%$ at the segmental level, respectively [42, 43]. Improved computed tomography (CT) scanners can also provide additional information, such as vascular wall thickness and assessment of lung parenchyma, mediastinal lymph nodes and bronchial arteries [20].

Typical CTEPH defects on CTPA include mosaic perfusion of lung parenchyma, organised eccentric thrombi lining proximal pulmonary vessels, large bronchial artery collaterals, variations in size of lobar and segmental vessels, complete or partial obstruction of pulmonary arteries, dilated central pulmonary arteries, right ventricular enlargement, filling defects, irregular vascular wall thickening, abrupt narrowing of vessels, and intraluminal webs or bands $[1,18,20]$. 
Sometimes acute PE may be difficult to differentiate from CTEPH but there are certain defects on CTPA which differ between the two conditions. Acute emboli appear as central intraluminal filling defects and, if located in an eccentric position, form an acute angle with the vessel wall, unlike in CTEPH where it would form an obtuse angle with the vessel wall. In addition, right ventricular enlargement and bronchial artery dilatation are unlikely defects observed with acute PE [44].

CTPA can lead to false-positive results as some conditions can mimic chronic thromboembolic disease, for example proximal lining thrombi associated with $\mathrm{PAH}$, pulmonary arteritis (e.g. Takayasu), tumour emboli into the pulmonary vasculature (commonly from breast, liver, renal and gastric carcinomas), fibrous mediastinitis or pulmonary artery sarcoma [44]. However, certain characteristic features of these conditions may be apparent on CTPA that enable an experienced clinician to distinguish their diagnoses from CTEPH.

CTPA can provide important information, complementary to pulmonary angiography, to assess the anatomical extent of surgically accessible CTEPH and evaluate surgical risk before PEA is performed. As CTPA assesses the mediastinum, it provides a useful evaluation of the lungs and aorta, and provides simple measurements such as right ventricular size and structure. It also has the advantage of assessing the lung parenchyma for coexisting respiratory pathology thereby providing a PEA risk assessment for CTEPH patients [20] and identifying bronchial artery dilatation, a potential complication for PEA that is prognostic for a poor surgical outcome [34].

CTPA has an advantage over conventional pulmonary angiography in that it does not require direct catheter access or the expertise that is needed to perform selective angiograms [14]. In specialist centres, CTPA may provide a reasonable noninvasive alternative to conventional pulmonary angiography for the evaluation of CTEPH $[14,34]$. However, it should be stressed that there are important limitations to using CTPA instead of $V^{\prime} / Q^{\prime}$ scanning for initial screening for CTEPH. As previously mentioned, compared with the $V^{\prime} / Q^{\prime}$ scan, CTPA has a lower sensitivity rate for CTEPH detection [36], is more difficult to interpret and is less likely to detect very distal disease forms. For the latter point, as disease in the very distal or subsegmental pulmonary arteries is less likely to be operable, the use of CTPA as a screening tool may limit the detection of patients with inoperable CTEPH who could be considered for medical therapy [14]. In addition, CTPA, similar to conventional pulmonary angiography, has the disadvantage of exposing the patient to radiation and nephrotoxic contrast agents in a cumulative manner given the numerous tests needed for diagnosis, surgical assessment and outcome monitoring.

The recent development of ECG-gated CTPA has permitted the simultaneous evaluation of thrombus location and right ventricular function, with good correlation between bowing of the intraventricular septum and systolic or mean PAP ( $r=-0.79$ and -0.86 , respectively; $\mathrm{p}<0.001)$ [43]. Further research is needed to determine if the use of this less invasive method may supersede conventional angiography and CTPA.

\section{New imaging approaches for CTEPH}

MRPA is used for morphological, anatomical and functional assessment of the heart and pulmonary circulation and offers considerable potential in CTEPH diagnosis, complementary to other imaging techniques [45]. Typical CTEPH defects with MRPA are similar to those seen with CTPA (e.g. intraluminal webs and bands, narrowing or obstruction of vessels, organised central thombi and dilated bronchial arteries) [46]. Contrast-enhanced MRPA has been shown to have a sensitivity of $83.1 \%$ and specificity of $98.6 \%$ in detecting thromboembolic material at the level of the main or lobar pulmonary arteries, and a sensitivity and specificity of $87.7 \%$ and $98.1 \%$, respectively, at the segmental level [47]. It has been suggested that phase-contrast MRPA is sufficient, in comparison with conventional pulmonary angiography, to identify pulmonary vascular occlusions [48], and has a similar accuracy to $V^{\prime} / Q^{\prime}$ scanning in diagnosing CTEPH but with better spatial resolution [45].

MRPA has the advantage of being a noninvasive technique with no radiation exposure. In addition to assessing the pulmonary vasculature, MRPA has the benefit of noninvasively assessing lung perfusion and right ventricular structure and function when combined with cine techniques [34]. Consequently, although magnetic resonance imaging (MRI) has not yet replaced pulmonary angiography in determining PAP and PVR, it can play a role in pre- and post-operative assessment of right ventricular function [17]. Contrast-enhanced MRPA is also used in specialist centres to provide a surgical "road map" in patients considered for PEA [34]. However MRPA has been shown to be inferior to CTPA beyond the segmental level [48] and a recent direct comparison between ECG-gated multi-detector CT angiography, MRPA and conventional pulmonary angiography suggested that ECG-gated multi-detector CT angiography was the superior method at all levels of the pulmonary vasculature [47]. Additionally, appropriate expertise is needed when using and interpreting data from this evolving technique.

In addition, other imaging modalities, such as DECTA, optical coherence tomography (OCT) and intravascular ultrasound (IVUS) are being evaluated in CTEPH. With DECTA, an iodine contrast medium 
is used and scans are taken at two different voltages to generate a map of parenchymal perfusion in addition to the standard anatomical CT images. It allows for the identification of perfusion defects distal to proximal vascular obstruction thereby providing enhanced detection of distal CTEPH over CTPA [49]. DECTA also improves vascular enhancement allowing assessment of bronchial artery collaterals. DECTA may, therefore, have a future role in simultaneously evaluating anatomy, perfusion and PEA suitability [49]. OCT is an interferometer-based optical imaging technique that produces a two-dimensional image of microstructures with a high resolution of 10-20 $\mu \mathrm{m}$ [50]. Initial data suggest that OCT may be useful in the differential diagnosis of distal CTEPH from PAH [50]. Finally, IVUS utilises a specially designed catheter with a miniaturised ultrasound probe to provide detailed images of the interior arterial walls in real time. IVUS has been used in conjunction with balloon pulmonary angioplasty, a novel potential therapeutic intervention for CTEPH, to determine the size of target lesions and therefore the optimal balloon size needed [16]. It was reported that the use of IVUS during this procedure resulted in a reduced risk of pulmonary artery rupture and reperfusion pulmonary injury, in addition to improved post-operative haemodynamic outcome.

Diagnostic pitfalls

Case 1: highlighting the importance of a $\mathrm{V}^{\prime} / \mathrm{Q}^{\prime}$ scan

A 58-year-old female presented with World Health Organization (WHO) functional class II/III PH and a 12-month history of dyspnoea on exertion [51]. Upon examination, clinical findings and the chest radiograph were unremarkable, while pulmonary function tests suggested marked hypoxaemia. The initial diagnosis was COPD and treatment with long-acting $\beta$-agonists was initiated; however, over a 2 -year period the patient's dyspnoea worsened, and she experienced syncope.

At this point, echocardiography was performed, revealing severe $\mathrm{PH}$ and right atrial and ventricular hypertrophy; Doppler systolic right ventricular pressure was $110 \mathrm{mmHg}$. A CT scan was performed, which showed no evidence of chronic thromboembolic disease.

RHC revealed extremely high PAP $(62 \mathrm{mmHg})$ but normal pulmonary capillary wedge pressure (7 mmHg). Cardiac output was $4.7 \mathrm{~L} \cdot \mathrm{min}^{-1}$ and PVR was $935 \mathrm{dyn} \cdot \mathrm{s} \cdot \mathrm{cm}^{-5}$. A diagnosis of idiopathic PAH was made and anticoagulation therapy plus bosentan was started. The patient's condition worsened and sildenafil was added without any improvement. At this point, a $V^{\prime} / Q^{\prime}$ scan was performed, which revealed bilateral subsegmental and segmental perfusion defects indicative of CTEPH. However, re-examination of the CT scan (taken with an old rather than a new multi-slice CT scanner) did not reveal any abnormalities that would have suggested the presence of chronic thromboembolic disease.

The patient then underwent pulmonary angiography, which showed large areas of the lungs that were not perfused due to thromboembolic obstructions. Finally, PEA was performed, after which the patient improved to WHO functional class I/II. Subsequent echocardiography revealed that the right ventricular diameter had returned to the near-normal range, highlighting the importance of $V^{\prime} / Q^{\prime}$ scanning and RHC in diagnosing CTEPH and assessing patient suitability for curative PEA surgery.

\section{Case 2: highlighting the consequences of misinterpretation of patient history}

A 68-year-old male presented with WHO functional class III PH and dyspnoea on exertion [18]. Initial investigations at his local hospital focused on ischaemic heart disease, and a coronary angiogram confirmed three-vessel disease. A coronary artery bypass graft was performed, which the patient survived after a prolonged stay in intensive care. However, the patient remained very breathless and a $V^{\prime} / Q^{\prime}$ scan was performed to exclude PE. This demonstrated mismatched perfusion defects. CTPA was carried out and CTEPH was confirmed. The patient was then referred to a specialist $\mathrm{PH}$ centre where RHC revealed a PAP of $50 \mathrm{mmHg}$ and a pulmonary capillary wedge pressure of $12 \mathrm{mmHg}$. Cardiac output was 5.1 L.min ${ }^{-1}$ and PVR was $680 \mathrm{dyn} \cdot \mathrm{s} \cdot \mathrm{cm}^{-5}$. An MRI pulmonary artery angiogram confirmed the CT findings, showing a web in the right upper lobe, bilateral lower lobe basal segmental webs and occlusion of the lingual artery. The patient underwent PEA, after which PAP decreased to $24 \mathrm{mmHg} ; 3$ months later he was WHO functional class I.

In this case the patient's original history was misinterpreted, and $\mathrm{PH}$ was not considered when investigations demonstrated ischaemic heart disease.

\section{Conclusions}

CTEPH should be considered in all patients with $\mathrm{PH}$ because early diagnosis helps to identify those patients suitable for potentially curative PEA. It is important that the condition is suspected in all at-risk patients, particularly those who have had previous PE, so that the correct diagnostic evaluations are performed in a timely manner. All patients with suspected CTEPH should be referred to a specialist centre for a complete diagnostic evaluation by a specialist multidisciplinary team to exclude or confirm the 
diagnosis, and assess suitability for PEA. Patients with CTEPH who are not candidates for surgery or have persistent/recurrent $\mathrm{PH}$ after PEA may be treatable with medical therapy, such as riociguat. Balloon pulmonary angioplasty is also emerging as a potential alternative treatment for selected inoperable patients with CTEPH.

Diagnosis of CTEPH generally requires input from several radiological techniques, including $V^{\prime} / Q^{\prime}$ scintigraphy, pulmonary angiography (conventional or CTPA) and haemodynamic evaluation by RHC. There is also increasing interest in the use of newer techniques, such as contrast-enhanced MRPA, DECTA, OCT and IVUS. As experience with these newer modalities increases, the current diagnostic algorithm may be updated, especially at experienced CTEPH centres.

\section{Acknowledgements}

Editorial assistance was provided by Adelphi Communications Ltd (Bollington, UK), supported by Bayer Pharma AG.

\section{References}

1 Hoeper MM, Madani MM, Nakanishi N, et al. Chronic thromboembolic pulmonary hypertension. Lancet Respir Med 2014; 2: 573-582.

2 Moser KM, Bloor CM. Pulmonary vascular lesions occurring in patients with chronic major vessel thromboembolic pulmonary hypertension. Chest 1993; 103: 685-692.

3 Frazier AA, Galvin JR, Franks TJ, et al. From the archives of the AFIP: pulmonary vasculature: hypertension and infarction. Radiographics 2000; 20: 491-524.

4 Becattini C, Agnelli G, Pesavento R, et al. Incidence of chronic thromboembolic pulmonary hypertension after a first episode of pulmonary embolism. Chest 2006; 130: 172-175.

5 Pengo V, Lensing AW, Prins MH, et al. Incidence of chronic thromboembolic pulmonary hypertension after pulmonary embolism. N Engl J Med 2004; 350: 2257-2264.

6 Bonderman D, Jakowitsch J, Adlbrecht C, et al. Medical conditions increasing the risk of chronic thromboembolic pulmonary hypertension. Thromb Haemost 2005; 93: 512-516.

7 Bonderman D, Wilkens $\mathrm{H}$, Wakounig S, et al. Risk factors for chronic thromboembolic pulmonary hypertension. Eur Respir J 2009; 33: 325-331.

8 Condliffe R, Kiely DG, Gibbs JS, et al. Improved outcomes in medically and surgically treated chronic thromboembolic pulmonary hypertension. Am J Respir Crit Care Med 2008; 177: 1122-1127.

9 Mayer E, Jenkins D, Lindner J, et al. Surgical management and outcome of patients with chronic thromboembolic pulmonary hypertension: results from an international prospective registry. J Thorac Cardiovasc Surg 2011; 141: 702-710.

10 Riedel M, Stanek V, Widimsky J, et al. Longterm follow-up of patients with pulmonary thromboembolism. Late prognosis and evolution of hemodynamic and respiratory data. Chest 1982; 81: 151-158.

11 Saouti N, Morshuis WJ, Heijmen RH, et al. Long-term outcome after pulmonary endarterectomy for chronic thromboembolic pulmonary hypertension: a single institution experience. Eur J Cardiothorac Surg 2009; 35: 947-952.

12 Dittrich HC, Nicod PH, Chow LC, et al. Early changes of right heart geometry after pulmonary thromboendarterectomy. J Am Coll Cardiol 1988; 11: 937-943.

13 D'Armini AM, Zanotti G, Ghio S, et al. Reverse right ventricular remodeling after pulmonary endarterectomy. J Thorac Cardiovasc Surg 2007; 133: 162-168.

14 Kim NH, Delcroix M, Jenkins DP, et al. Chronic thromboembolic pulmonary hypertension. J Am Coll Cardiol 2013; 62: Suppl., D92-D99.

15 Hoeper MM. Pharmacological therapy for patients with chronic thromboembolic pulmonary hypertension. Eur Respir Rev 2015; 24: 272-282.

16 Mizoguchi H, Ogawa A, Munemasa M, et al. Refined balloon pulmonary angioplasty for inoperable patients with chronic thromboembolic pulmonary hypertension. Circ Cardiovasc Interv 2012; 5: 748-755.

17 Giannouli E, Maycher B. Imaging techniques in chronic thromboembolic pulmonary hypertension. Curr Opin Pulm Med 2013; 19: 562-574.

18 Jenkins D, Mayer E, Screaton N, et al. State-of-the-art chronic thromboembolic pulmonary hypertension diagnosis and management. Eur Respir Rev 2012; 21: 32-39.

19 Galiè N, Hoeper MM, Humbert M, et al. Guidelines for the diagnosis and treatment of pulmonary hypertension. Eur Respir J 2009; 34: 1219-1263.

20 Auger WR, Kerr KM, Kim NH, et al. Evaluation of patients with chronic thromboembolic pulmonary hypertension for pulmonary endarterectomy. Pulm Circ 2012; 2: 155-162.

21 Bonderman D, Turecek PL, Jakowitsch J, et al. High prevalence of elevated clotting factor VIII in chronic thromboembolic pulmonary hypertension. Thromb Haemost 2003; 90: 372-376.

22 Wolf M, Boyer-Neumann C, Parent F, et al. Thrombotic risk factors in pulmonary hypertension. Eur Respir J 2000; 15: 395-399.

23 Reesink HJ, Tulevski II, Marcus JT, et al. Brain natriuretic peptide as noninvasive marker of the severity of right ventricular dysfunction in chronic thromboembolic pulmonary hypertension. Ann Thorac Surg 2007; 84: 537-543.

24 Klok FA, Tesche C, Rappold L, et al. External validation of a simple non-invasive algorithm to rule out chronic thromboembolic pulmonary hypertension after acute pulmonary embolism. Thromb Res 2014 [In press DOI: 10.1016/j.thromres.2014.12.009].

25 Lankeit M, Dellas C, Panzenböck A, et al. Heart-type fatty acid-binding protein for risk assessment of chronic thromboembolic pulmonary hypertension. Eur Respir J 2008; 31: 1024-1029.

26 Naito A, Tanabe N, Jujo T, et al. Pentraxin3 in chronic thromboembolic pulmonary hypertension: a new biomarker for screening from remitted pulmonary thromboembolism. PLoS One 2014; 9: e113086.

27 Quarck R, Nawrot T, Meyns B, et al. C-reactive protein: a new predictor of adverse outcome in pulmonary arterial hypertension. J Am Coll Cardiol 2009; 53: 1211-1218. 

cteph-association.org/meetings/paris-2014/abstracts-icc-2014/ the task force for the diagnosis and treatment of pulmonary hypertension of the European Society of Cardiology (ESC) and the European Respiratory Society (ERS), endorsed by the International Society of Heart and Lung Transplantation (ISHLT). Eur Heart J 2009; 30: 2493-2537.

31 Raisinghani A, Ben-Yehuda O. Echocardiography in chronic thromboembolic pulmonary hypertension. Semin Thorac Cardiovasc Surg 2006; 18: 230-235.

32 Surie S, Gibson NS, Gerdes VE, et al. Active search for chronic thromboembolic pulmonary hypertension does not appear indicated after acute pulmonary embolism. Thromb Res 2010; 125: e202-e205.

33 Mehta S, Helmersen D, Provencher S, et al. Diagnostic evaluation and management of chronic thromboembolic pulmonary hypertension: a clinical practice guideline. Can Respir J 2010; 17: 301-334.

34 Coulden R. State-of-the-art imaging techniques in chronic thromboembolic pulmonary hypertension. Proc Am Thorac Soc 2006; 3: 577-583.

35 Fishman AJ, Moser KM, Fedullo PF. Perfusion lung scans vs pulmonary angiography in evaluation of suspected primary pulmonary hypertension. Chest 1983; 84: 679-683.

36 Tunariu N, Gibbs SJ, Win Z, et al. Ventilation-perfusion scintigraphy is more sensitive than multidetector CTPA in detecting chronic thromboembolic pulmonary disease as a treatable cause of pulmonary hypertension. $J$ Nucl Med 2007; 48: 680-684.

37 Bergin CJ, Sirlin CB, Hauschildt JP, et al. Chronic thromboembolism: diagnosis with helical CT and MR imaging with angiographic and surgical correlation. Radiology 1997; 204: 695-702.

38 Wartski M, Collignon MA. Incomplete recovery of lung perfusion after 3 months in patients with acute pulmonary embolism treated with antithrombotic agents. THESEE Study Group. Tinzaparin ou Heparin Standard: Evaluation dans l'Embolie Pulmonaire Study. J Nucl Med 2000; 41: 1043-1048.

39 Ryan KL, Fedullo PF, Davis GB, et al. Perfusion scan findings understate the severity of angiographic and hemodynamic compromise in chronic thromboembolic pulmonary hypertension. Chest 1988; 93: 1180-1185.

40 McLaughlin VV, Langer A, Tan M, et al. Contemporary trends in the diagnosis and management of pulmonary arterial hypertension: an initiative to close the care gap. Chest 2013; 143: 324-332.

41 Auger WR, Kim NH, Trow TK. Chronic thromboembolic pulmonary hypertension. Clin Chest Med 2010; 31: 741-758.

42 Reichelt A, Hoeper MM, Galanski M, et al. Chronic thromboembolic pulmonary hypertension: evaluation with 64-detector row CT versus digital substraction angiography. Eur J Radiol 2009; 71: 49-54

43 Sugiura T, Tanabe N, Matsuura Y, et al. Role of 320-slice CT imaging in the diagnostic workup of patients with chronic thromboembolic pulmonary hypertension. Chest 2013; 143: 1070-1077.

44 Wijesuriya S, Chandratreya L, Medford AR. Chronic pulmonary emboli and radiologic mimics on CT pulmonary angiography: a diagnostic challenge. Chest 2013; 143: 1460-1471.

45 Rajaram S, Swift AJ, Telfer A, et al. 3D contrast-enhanced lung perfusion MRI is an effective screening tool for chronic thromboembolic pulmonary hypertension: results from the ASPIRE Registry. Thorax 2013; 68: 677-678

46 Kreitner KF, Kunz RP, Ley S, et al. Chronic thromboembolic pulmonary hypertension - assessment by magnetic resonance imaging. Eur Radiol 2007; 17: 11-21.

47 Ley S, Ley-Zaporozhan J, Pitton MB, et al. Diagnostic performance of state-of-the-art imaging techniques for morphological assessment of vascular abnormalities in patients with chronic thromboembolic pulmonary hypertension (CTEPH). Eur Radiol 2012; 22: 607-616.

48 Ley S, Kauczor HU, Heussel CP, et al. Value of contrast-enhanced MR angiography and helical CT angiography in chronic thromboembolic pulmonary hypertension. Eur Radiol 2003; 13: 2365-2371.

49 Hoey ET, Mirsadraee S, Pepke-Zaba J, et al. Dual-energy CT angiography for assessment of regional pulmonary perfusion in patients with chronic thromboembolic pulmonary hypertension: initial experience. $A J R A m J$ Roentgenol 2011; 196: 524-532.

50 Tatebe S, Fukumoto Y, Sugimura K, et al. Optical coherence tomography as a novel diagnostic tool for distal type chronic thromboembolic pulmonary hypertension. Circ J 2010; 74: 1742-1744.

51 Hoeper MM, Laenger F. Challenging cases in PH. Eur Respir Rev 2007; 16: 19-23. 